\title{
La visibilidad de la autoría en el videoclip post-televisivo: La dignificación del vídeo musical a través de la reivindicación de los creadores en los créditos
}

\section{Resumen}

Los objetivos principales de esta investigación son analizar el modo en que se identifica la autoría en los vídeos musicales e indagar en las razones por las que se reconoce dicha autoría desde el propio discurso. Consideramos que los títulos de crédito en el videoclip se han convertido en una práctica habitual. Se produce de esta forma un cambio de paradigma con respecto a la atribución de autoría del videoclip, previamente oculta. Planteamos como hipótesis principal que la visibilidad de la autoría en el vídeo musical va más allá de la mera transmisión de información, relativa a la producción, e implica una reivindicación de la figura de los creadores, como marca o muestra de identidad en el discurso. La reivindicación de la autoría supondría así una forma de dignificar el videoclip como formato de relieve no sólo a nivel industrial y comercial, sino también artístico. Con objeto de demostrar esta hipótesis, hemos analizado los videoclips de los 40 singles más populares en el Reino Unido en 2017, según la consultora The Official Charts. El análisis de la presencia de información sobre la autoría de las piezas analizadas ofrece resultados variados que no permiten contrastar ninguna tendencia mayoritaria o abiertamente consolidada, pero sí permite advertir prácticas frecuentes que podrían ser representativas del estado actual del vídeo musical. La visibilidad de la autoría permite al formato una consideración más cercana a la del producto cultural o a la creación artística, disimulando su función comercial. El videoclip no abandona dicho objetivo comercial o promocional, ligado a su propia naturaleza como formato, pero potencia su naturaleza de creación artística.

\author{
José Patricio Pérez Rufí. Phd \\ Doctor en Comunicación \\ Audiovisual \\ Profesor Universidad de Málaga \\ Málaga, España \\ Correo electrónico: \\ patricioperez@uma.es \\ ๑ orcid.org/0000-0002-7084-3279 \\ Google Scholar
}

Recibido: julio 3 de 2018

Aprobado: junio 07 de 2019

\section{Palabras clave:}

Análisis del discurso audiovisual, teoría del autor, títulos de crédito, videoclip, YouTube. 


\section{The visibility of the authorship in the post-television video clip: the dignification of the music video through the claim of the creators in the credits}

\begin{abstract}
The aims of this paper are to analyze how authorship is identified in music videos and to inquire the reasons why this authorship is recognized from the discourse itself. The video clips credit titles are considered to have become a standard practice and in this way, a change of paradigm in relation to the attribution of authorship in the music video, previously hidden, occurs. The main hypothesis proposed is that the visibility of the authorship in the music video go beyond the transmission of information related to production and implies a vindication of the creators, as a brand or sample of identity in the discourse. The vindication of authorship would then suppose a way to dignify the video clip as an important format not only at the industrial and commercial level, but also at an artistic level. In order to demonstrate this hypothesis, the music videos of the 40 most popular singles in the United Kingdom in 2017, according to The Official Charts consultancy company, were analyzed. The analysis of the presence of information about the authorship in the analyzed pieces offers varied results that do not allow confirming any majority or openly consolidated trend, but it does allow identifying frequent practices that could be representative of the current situation of the video clip. The visibility of authorship allows the format a closer consideration to that of the cultural product or artistic creation, disguising its commercial function. The video clip does not abandon its commercial or promotional objective linked to its own nature as a format, but it enhances its nature of artistic creation.
\end{abstract}

Key words:

Audiovisual analysis, auteur theory, video clips, opening titles, YouTube. 


\section{Introducción}

Frente a otros discursos como el cinematográfico o el televisivo, en los que la autoría se recoge textualmente como un factor capaz de dotar al producto de identidad, el videoclip en sus primeras décadas de historia ha visto ignorado este reconocimiento y en muy pocas ocasiones se ha incluido en el propio discurso la atribución de la autoría. Este hecho se explica desde la misma indiferencia con que se atendía a la identificación de los creadores de estas piezas, como anónimos realizadores al servicio de los intérpretes musicales, que simbólicamente pasaban a responsabilizarse de todos los aspectos creativos del vídeo musical, incluso si esto no era así.

En la década de los ochenta hubo excepciones a la ocultación de la autoría del videoclip y estuvieron ligadas a célebres realizadores que hacían incursiones puntuales en el campo del videoclip, caso de John Landis o Martin Scorsese en sus trabajos para Michael Jackson o de otros como Spike Lee (Public Enemy, Prince), Brian de Palma (Bruce Springteen), Jonathan Demme (UB40, New Order), David Fincher (iniciado en el videoclip antes de emprender su carrera como director de largometrajes, con clips para Madonna, Sting, George Michael o Aerosmith), Gus Van Sant (Red Hot Chili Peppers, David Bowie) o Michael Bay (Meat Loaf).

Sin embargo, es en la década de los noventa cuando surgen varios directores de videoclips que adquieren la categoría de realizadores estrella del formato o "autores-clip", como los define Rodríguez-López (2017), entre los que se encontraría Michel Gondry, Chris Cunningham o Spike Jonze, además de otros muchos que ya estaban en la órbita del videoclip con una fuerte identidad proyectada en sus creaciones, como Jean-Baptiste Mondino o Anton Corbijn. Podríamos afirmar que este grupo de directores, apoyados por las ediciones videográficas de sus trabajos y algunas publicaciones monográficas 
que reivindicaban la importancia de su figura y su obra, conforma la primera generación específica de directores de videoclips con un reconocimiento autoral.

Con la distribución online del videoclip y YouTube, Vevo o Vimeo como principales plataformas de acceso a aquellos, el vídeo musical entra en una etapa en la que se acelera la experimentación al liberarse de los condicionantes formales y de contenidos que imponía la producción previa para televisión, al tiempo que se introducen nuevas tendencias, propagadas con rapidez, que convierten al videoclip en un formato en permanente evolución y capaz aún de sorprender y sobrepasar límites que ahora no tienen sentido de ser y que llegan a cuestionar la naturaleza del formato musical comercial y sus objetivos últimos.

Consideramos que, entre estas tendencias, se encuentra una reivindicación de la autoría del videoclip que va mucho más allá de los directores estrella y que alcanza a muchos creadores en diferentes disciplinas, no siempre de evidente notoriedad. Esta visibilidad de la identificación autoral se hace desde el propio discurso, a través de textos (extradiegéticos) sobreimpresos en pantalla que indican los nombres de algunos responsables de la pieza. En algunos casos se identificará únicamente al intérprete musical o el título de la canción, una información que ya se ofrecía en los vídeos emitidos por programas de televisión.

Creemos que en los últimos años estos textos también han recogido el nombre de la persona responsable de la realización, pero igualmente otro tipo de información relevante capaz de dotar de identidad al discurso como la productora, la dirección de fotografía, la dirección artística o actores y actrices, modelos o personas participantes en el campo de la actuación. Esta tendencia nace desde la ruptura de los límites de la programación televisiva lineal, dado 
que en las plataformas de vídeo online las duraciones de las piezas no vienen condicionadas por la emisión lineal y se cuenta con una mayor libertad en este sentido, ahora dependientes de la voluntad del usuario. Consideramos que estos textos discursivos constituyen una forma de proto-títulos de créditos con la misma función informativa con que aparecen en largometrajes, cortometrajes, series de televisión o programas de televisión, en general. Planteamos como hipótesis principal que la visibilidad de la autoría en el vídeo musical va más allá de la mera transmisión de información relativa a la producción e implica una reivindicación de la figura de los creadores, como marca o muestra de identidad en el discurso. La reivindicación de la autoría supondría así una forma de dignificar el videoclip como formato de relieve no sólo a nivel industrial y comercial, sino también artístico.

Los objetivos principales de esta investigación son analizar el modo en que se identifica la autoría en los vídeos musicales e indagar en las razones por las que se reconoce dicha autoría desde el propio discurso. Esta posible tendencia, cuya presencia requeriría de una constatación previa y justificada, quedaría ligada al actual contexto post-televisivo de producción y difusión de contenidos audiovisuales, que podría convivir con la persistencia de prácticas habituales en la televisión lineal.

La literatura académica previa acerca de una cuestión tan precisa, a la que Vernallis (2008) se refiere como "la cuestión del autor" en el videoclip, es muy reducida, si bien encontramos los textos en castellano de Viñuela (2008) o Rodríguez-López (2017), además de trabajos como los de Reiss y Feineman (2000), Hanson (2006), Fidler (2007) o Keazor y Wübbena (2010). Sin embargo, enlaza con la extensa bibliografía relativa a la Politique des Autors o Auteur Theory. Este trabajo pretende actualizar las investigaciones relacionadas con la autoría en el vídeo musical a partir del análisis de contenido de una muestra representativa de prácticas recientes. 


\section{El videoclip post-televisivo: la distribución online lo cambia todo}

El vídeo musical depende de las condiciones extradiscursivas en las que se produce y se difunde. Si bien la televisión lineal se mantiene como vía de distribución del videoclip, actualmente la distribución prioritaria se hace sobre todo mediante portales de vídeo online como YouTube o Vimeo (Pérez Rufí, 2017, p. 577).

En 2005 nace YouTube y se erige con rapidez como la principal plataforma de vídeo online. Incluso si su supuesta democratización se cuestiona (DeAguilera-Moyano, Castro-Higueras \& Pérez-Rufí, 2019) y a pesar de no ser la primera ni la única plataforma con objetivos similares, se ha convertido en el referente contemporáneo de distribución y consumo masivos del videoclip. La reducción de presupuestos que implicó la digitalización de los medios de registro y la participación del usuario en la creación y la interacción con los contenidos consolidaron a YouTube como "plataforma de videoclips", Ilegando a desplazar a la mítica MTV de su identidad nativa. Viñuela (2013) Ilega a definir YouTube como "la nueva MTV del siglo XXI" (p. 170). Según Tarín Cañadas (2013), "si anteriormente, su soporte era principal la televisión, para la que nació, ahora su campo de acción es Internet (p. 8)". El vídeo musical, apunta Sedeño (2010), se desplaza hacia los medios digitales online, como nuevos territorios para su distribución.

El empleo del medio Internet como canal de difusión de videoclips "tiene repercusiones sobre numerosas dimensiones de estos, como sus propios aspectos formales" (Selva, 2012), además de generar "un escenario muy diferente" que afecta a lo formal y a "su funcionamiento como herramienta de comunicación comercial".

En la evolución de esta idea, y tomando en consideración la argumentación de Vernallis (2013), para quien el vídeo se encuentra en una nueva era de 
su historia, Sedeño, Rodríguez y Roger (2016) afirman que la naturaleza del formato de promoción musical viene condicionada por una industria que se encuentra en continuo cambio y transformación con escaso interés por su archivo o su conservación, en "lo que podría denominarse videoclip postelevisivo".

Consecuentemente, "el videoclip ha dejado de ser un producto secundario en una sociedad cada día más audiovisual en la que cada vez hay más pantallas en las que el videoclip es un contenido habitual" (Viñuela, 2013, p. 170). La importancia del número de reproducciones de vídeos musicales en la célebre lista Billboard desde 2013 condujo a que las discográficas ofrecieran una mayor representación de sus contenidos audiovisuales promocionales en portales como YouTube o Vevo. Sostiene Subires Mancera (2012) que, si bien en un principio las discográficas parecían ver con malos ojos fenómenos como YouTube, "finalmente acabaron dándose cuenta del enorme potencial promocional que tenían (p. 1612)", dado que el videoclip es "una potente herramienta de promoción del artista y de su obra, independientemente de su propia identidad como creación audiovisual".

La distribución a través de Internet permite liberar al vídeo musical de la televisión y de todo lo que ello implica. De entrada, esto significa que el videoclip no se integra dentro de una cadena lineal de contenidos decidida por los editores de la emisora. La ruptura de la linealidad tiene una consecuencia directa en su posible duración o en la articulación de estrategias narrativas transmedia (Pérez Rufí, 2017, p. 585). El visionado ya no tiene por qué ser lineal ni completarse. La independencia con respecto a la televisión permite además una mayor libertad al videoclip, en cuanto a contenidos, siempre condicionados por la financiación por parte de una industria, la discográfica, en fuerte crisis a partir del cambio de siglo. 
Al volver a la cuestión de la autoría, señala Viñuela (2008) que el anonimato del director de videoclips "forma parte de una estrategia comercial, equiparable a lo que fue el studio system en la industria del cine estadounidense" (p. 244), en el sentido de que concentraba la atención en el producto o el artista y obviaba elementos prescindibles para su promoción, como es la responsabilidad de la dirección.

En el modelo tradicional del videoclip, el director "pocas veces ve reconocida la autoría de su trabajo y casi nunca existen unos títulos de crédito", de donde surge una invisibilidad de la autoría ligada a la estrategia comercial del vídeo, centrada en la figura del intérprete, percibido como "responsable último de todas las fases y productos que estén relacionados con su figura" (Viñuela, 2013, pp. 172-173). De aquí resulta la ausencia de estudios en torno a la autoría en el clip musical señalada por Vernallis (2013), incluso si el concepto de estilo puede Ilegar a ser más fuerte en un videoclip que en un filme.

Fidler (2008) apunta al propio formato del videoclip como responsable de la ocultación del director, en el sentido de que el vídeo musical tiene una función comercial y no es el medio sobre el que el director base su carrera, sino que suele tratarse de un punto de transición hacia los largometrajes de ficción. Actualmente, un amplio número de directores se ocupa en el videoclip de forma simultánea a otros formatos (por lo general, publicitarios), pero el objetivo último no implica necesariamente al largometraje.

La teoría del autor (o Politique des Auteurs) Ilevaría a los críticos de Cahiers du Cinéma a reivindicar la figura del director de cine como personalidad artística capaz de dotar a la obra de la impronta y la identidad del creador, de forma similar a otras disciplinas artísticas. Esta noción del autor, argumentan Higueras-Ruiz, Gómez-Pérez y Alberich-Pascual (2019), es susceptible de aplicación también a las producciones televisivas de ficción, incluso si se 
tratan de procesos colectivos, como un estilo creativo personal en el conjunto de su obra audiovisual.

En el caso del videoclip, según Viñuela (2013), el habitual anonimato del director convive "con una tendencia opuesta que otorga un mayor protagonismo al director que al artista" (p. 172). Este aspecto introduce una variante importante con respecto a la noción de autoría de la obra. Sedeño (2012) afirma que el clip sigue así una tradición anti-autoral: "habitualmente, el artista queda en el imaginario del fan como el 'autor' del vídeo, y sus directores reales pertenecen a la trastienda de la industria, excepto casos muy conocidos como Stephane Sednaoui, Chris Cunningham, Michel Gondry y otros" (p. 1228). En el caso del reconocimiento autoral "el prestigio y el reconocimiento social del director se traslada al artista protagonista del videoclip" (Viñuela, 2013, p. 172).

Rodríguez-López (2017) defiende la existencia de un videoclip de autor contrapuesta al videoclip (convencional, o de estudio), "del mismo modo que el cine de autor es opuesto al cine comercial", como consecuencia de la distribución online y del reconocimiento por parte del usuario-espectador, ahora con información y contenidos que permiten al videófilo identificar, analizar, relacionar e interpretar "la obra del director clip del mismo modo que sucede con los films de directores de cine prestigiosos" (p. 239).

A través del análisis de la videografía de dos directores tan dispares como Francis Lawrence y Dave Meyers, Vernallis (2013) concluye que lo más revelador es que un video musical proporciona un registro de las relaciones de un director con una canción (p. 261), emergiendo el estilo (fugazmente) en las relaciones entre música, imagen y texto. Es en esta relación con la canción desde la que podría emerger la noción de autoría, pese a la dificultad que intrinca su reconocimiento. 
La autoría se convierte de esta forma en una "marca de estilo", es decir, "una huella personal que el autor aplica sobre la obra audiovisual, la cual suele ser reiterativa durante su trayectoria laboral" (Higueras-Ruiz et al., 2019, p. 92). Hablamos, en definitiva, de marca e identidad: la obra queda configurada y condicionada desde la perspectiva de los creadores, al punto de que los intérpretes y las discográficas pueden demandar su aplicación en las piezas que crearán y de que la audiencia podría reconocer dichas claves estilísticas asociadas a los creadores. Rodríguez-López (2017) va más allá al afirmar que "el estilo de cada creativo traspasa las propias características del formato del videoclip para transmitir al espectador un universo personal e íntimo (p. 238)".

Las herramientas de creación y distribución 2.0 también permiten compartir la autoría a través de la creación en cadena o simultánea de varios usuarios. La implicación del usuario, esta vez el fan, lo lleva a ser directamente el productor del mismo, quedando la filmación de manos de aquellos y la edición de parte de la discográfica. Sería, por ejemplo, el caso de "Maroon 5 - Daylight" (2012), como proyecto dirigido por Jonas Åkerlund en el que la banda no aparece y en su lugar se editan los planos rodados por sus seguidores. Otro caso podría ser el de "Happy" de Pharrel Williams, en el que el músico invitó a los usuarios a crear un videoclip a medio camino entre el vídeo promocional turístico y el de valorización de la identidad local de los vecinos de diversas ciudades, en los primeros meses de 2014. Posponemos, en cualquier lugar, el estudio de la producción colectiva de vídeos musicales para una investigación posterior, dada la necesidad previa de análisis de la visibilidad de la misma autoría, objeto principal de este trabajo.

\section{Metodología}

Hemos tomado como muestra para este análisis el chart de los 40 sencillos más populares de 2017 elaborado por The Official Charts Company (2018). Este 
listado contempla tanto el número de ventas de cada uno de los singles a lo largo del 2017 como el número de reproducciones de diferentes plataformas de música y vídeo en streaming. The Official Charts Company es una joint venture formada por BPI (British Phonographic Industry) y Entertainment Retailers Association, que, de la mano de la consultora Millward Brown, publica con regularidad semanal el listado de los singles y álbumes más populares en el Reino Unido, con productos como Official Singles Charts, Official Albums Charts o el sitio web OfficialCharts.com.

La razón por la que acudimos a este listado se encuentra en la necesidad de atención a una muestra objetiva no ligada a los valores estéticos o artísticos de los videoclips o de los singles. Elegimos The Official Singles Charts de Reino Unido frente a otros charts como Billboard o las listas de contenidos musicales más populares en YouTube por la mayor variedad estilística ofrecida, dado que el actual mercado doméstico estadounidense está dominado por el hip hop y el trap y otros mercados nacionales, como el español, por géneros como el reguetón o el trap. Entendemos que atender a un solo género musical habría sesgado y condicionado en exceso los resultados.

Dentro de esta muestra, recogemos en primer lugar el nombre de la pieza analizada y su duración. A continuación, observamos la presencia de textos informando de la autoría en el propio discurso pero también en el texto que acompaña a cada vídeo en su página de YouTube, que contextualizaría el contenido.

Tomamos nota de si estos textos presentes en el discurso mencionan al intérprete, a la persona responsable de la dirección, el estudio productor y otro tipo de personal creativo. Categorizamos como "información contextual" los textos que no pertenecen al discurso audiovisual pero que sí forman parte de la información ofrecida por YouTube, que en algunos casos identificarán 
la autoría de la pieza (realización y todo tipo de personal implicado en la producción).

Apuntamos el número de videoclips (videografía) de los que tengamos constancia según la base de datos Imvdb.com, dirigidos por los realizadores identificados. Indicamos entre paréntesis el nombre del realizador y el número de videoclips en aquellos casos en los que el nombre no aparece como texto inserto en el discurso, sino en la página de YouTube.

Proponemos en el siguiente punto un análisis de contenido de los propios créditos y de la composición tipográfica en los se menciona la autoría, adaptando la metodología de Pérez Rufí (2018). Identificamos en primer lugar dentro del discurso si los textos son diegéticos o extradiegéticos (Casetti \& Di Chio, 1991). Los textos diegéticos pertenecen al plano de la historia (han sido filmados como elemento integrado dentro de la puesta en escena), mientras que los textos no diegéticos (o extradiegéticos) no pertenecen a la puesta en escena y son incorporados en la edición de la pieza.

Dentro de este mismo apartado, atenderemos al movimiento de los textos, es decir, si se tratan de tipografías cinéticas, así como al tamaño de los textos con los nombres de los autores en relación con el tamaño del cuadro o frame: consideraremos que el texto tiene un tamaño grande si éste ocupa alrededor del $50 \%$ de la superficie o más; un tamaño medio ocuparía entre el 40 y el $20 \%$ de la superficie; y un tamaño pequeño ocuparía un espacio inferior al $20 \%$ del frame. Estos valores son arbitrarios pero nos permiten aplicarlos sobre toda la muestra de una manera uniforme para llegar a resultados coherentes.

En cuarto lugar, dentro del análisis de la composición tipográfica, las clasificamos para intentar descubrir tendencias al respecto. Son muchas las 
clasificaciones de familias tipográficas, pero aplicaremos por su simplicidad y facilidad en el reconocimiento de los tipos la propuesta de Robin Williams (2015), a partir de la combinación y simplificación de otras clasificaciones. Williams distingue seis categorías: Oldstyle (tipografías con serifa con remates en ángulo, transición grueso/fino moderado y distribución diagonal de partes más finas), Modern (tipografías con serifa con remate horizontal delgado, transición grueso/fino radical y acento vertical), Slab Serif (tipografías con serifa con remate horizontal grueso, sin apenas transición grueso/fino y acento vertical), Sans Serif (tipografías sin serifa), Script (tipografías que imitan la escritura manual a través de cualquier instrumento) y decorativas (todas las demás fuentes que no siguen los parámetros de las categorías anteriores).

Una vez realizado en el análisis y antes de la discusión de resultados, a partir de los nombres recogidos en la muestra, destacaremos los autores más relevantes por número de videoclips dirigidos según la base de datos Imvdb. com. Esta breve mención a los responsables de la dirección obedece no tanto a la voluntad de hacer un estudio desde una perspectiva estética, objetivo que no tiene esta investigación, como a la necesidad de concretar la mayor visibilidad de la autoría en el propio videoclip en creadores precisos.

\section{Análisis}

Recogemos en la siguiente tabla únicamente el análisis de aquellas piezas que incluyen alguna mención a la autoría o responsabilidad de alguna de las facetas creativas del vídeo musical, ya sea en el propio discurso audiovisual o en la "información contextual" de la página en YouTube. Serían un total de 31 vídeos, es decir, el 77,5\% de la muestra. 
Tabla 1. Análisis de la presencia de información acerca de la autoría en los videoclips del Chart 40 de Reino Unido del año 2017.

Elaboración propia.

\begin{tabular}{|c|c|c|c|c|c|c|c|c|c|c|}
\hline \multirow[t]{2}{*}{ Vídeo } & \multirow[t]{2}{*}{ Durac } & \multicolumn{4}{|c|}{ Visibilidad de la autoría (recogido en textos) } & \multirow[t]{2}{*}{ Vídeo-grafía } & \multicolumn{4}{|c|}{ Análisis tipográfico } \\
\hline & & Intér & Dirección & $\begin{array}{l}\text { Personal/ } \\
\text { Producción }\end{array}$ & Contex & & $\begin{array}{l}\text { Dieg } \\
\text { Extrad. }\end{array}$ & Cinética & Tamaño & Categoría \\
\hline $\begin{array}{l}\text { Ed Sheeran - Shape of You } \\
\text { [Official Video] }\end{array}$ & $04: 23$ & $x$ & Jason Koenig & Actores & $\mathrm{x}$ & 14 & Extrad & No & Medio & Sans serif \\
\hline $\begin{array}{l}\text { Ed Sheeran - Castle On The } \\
\text { Hill [Official Video] }\end{array}$ & $04: 48$ & & $\begin{array}{l}\text { (George } \\
\text { Belfield) }\end{array}$ & & $x$ & (9) & & & & \\
\hline $\begin{array}{l}\text { French Montana } \\
\text { Unforgettable ft. Swae Lee }\end{array}$ & $04: 46$ & $x$ & $\begin{array}{l}\text { Spiff Tv } \\
\text { y French } \\
\text { Montana }\end{array}$ & Productora & & 5 y 1 & Extrad & No & Grande & Modern \\
\hline $\begin{array}{l}\text { Ed Sheeran - Galway Girl } \\
\text { [Official Video] }\end{array}$ & 03:19 & $x$ & Jason Koenig & $\begin{array}{l}\text { Actriz, } \\
\text { operador } \\
\text { de cámara }\end{array}$ & $x$ & 14 & Extrad & No & Medio & Modern \\
\hline $\begin{array}{l}\text { Ed Sheeran - Perfect } \\
\text { (Official Music Video) }\end{array}$ & 04:39 & & Jason Koenig & Actriz & $x$ & 14 & Extrad & No & Grande & Script \\
\hline $\begin{array}{l}\text { The Chainsmokers \& } \\
\text { Coldplay - Something Just } \\
\text { Like This (Lyric) }\end{array}$ & 04:07 & $x$ & $\begin{array}{l}\text { James } \\
\text { Zwadlo }\end{array}$ & & & 14 & Extrad & Sí & Grande & Decorativa \\
\hline $\begin{array}{l}\text { Jax Jones - You Don't Know } \\
\text { Me (Official Video) ft. RAYE }\end{array}$ & 03:31 & $x$ & & & & & Extrad & No & Grande & $\begin{array}{l}\text { Decorativa, } \\
\text { Sans serif }\end{array}$ \\
\hline $\begin{array}{l}\text { Dua Lipa - New Rules } \\
\text { (Official Music Video) }\end{array}$ & 03:44 & $x$ & & & & & Extrad & Sí & Medio & Sans serif \\
\hline $\begin{array}{l}\text { DJ Khaled - I'm The One } \\
\text { ft. Justin Bieber, Quavo, } \\
\text { Chance the Rapper, Lil } \\
\text { Wayne }\end{array}$ & $05: 21$ & $x$ & Eif Rivera & $\begin{array}{l}\text { Productor } \\
\text { ejecutivo }\end{array}$ & & 57 & $\begin{array}{l}\text { Dieg/ } \\
\text { Extrad }\end{array}$ & No & $\begin{array}{l}\text { Grande } \\
\text { Pequeño }\end{array}$ & $\begin{array}{l}\text { Oldstyle, } \\
\text { Sans serif }\end{array}$ \\
\hline $\begin{array}{l}\text { DJ Khaled - Wild Thoughts } \\
\text { ft. Rihanna, Bryson Tiller }\end{array}$ & 03:35 & $x$ & Colin Tilley & & & 128 & Extrad & Sí & Grande & $\begin{array}{l}\text { Sans serif, } \\
\text { Script }\end{array}$ \\
\hline $\begin{array}{l}\text { Jonas Blue - Mama ft. } \\
\text { William Single }\end{array}$ & 03:03 & $x$ & & & & & Extrad & No & Medio & Sans serif \\
\hline $\begin{array}{l}\text { Martin Jensen - Solo Dance } \\
(4 \mathrm{~K})\end{array}$ & $03: 33$ & $x$ & $\begin{array}{l}\text { Nicolas } \\
\text { Tobias } \\
\text { Følsgaard }\end{array}$ & $\begin{array}{l}\text { Productora, } \\
\text { coreogr., } \\
\text { fotogr., } \\
\text { actores } \\
\end{array}$ & & 49 & Extrad & No & Pequeño & Sans serif \\
\hline $\begin{array}{l}\text { Shawn Mendes - There's } \\
\text { Nothing Holdin' Me Back }\end{array}$ & $03: 57$ & $\mathrm{x}$ & & Actriz & & & Extrad & No & Grande & Sans serif \\
\hline $\begin{array}{l}\text { Little Mix - Touch (Official } \\
\text { Video) }\end{array}$ & $03: 37$ & $x$ & $\begin{array}{l}\text { Director } \\
\text { X y Parris } \\
\text { Goebel }\end{array}$ & & & $\begin{array}{l}209 \\
3\end{array}$ & Extrad & Sí & Grande & $\begin{array}{l}\text { Decorativa, } \\
\text { Sans serif }\end{array}$ \\
\hline $\begin{array}{l}\text { Camila Cabello - Havana } \\
\text { ft. Young Thug }\end{array}$ & $06: 42$ & $x$ & $\begin{array}{l}\text { Dave } \\
\text { Meyers }\end{array}$ & Actores & & 163 & $\begin{array}{l}\text { Extrad } \\
\text { Dieg } \\
\end{array}$ & No & Medio & $\begin{array}{l}\text { Script, sans } \\
\text { serif }\end{array}$ \\
\hline $\begin{array}{l}\text { Jason Derulo - Swalla } \\
\text { (feat. Nicki Minaj \& Ty } \\
\text { Dolla \$ign) (Official Music } \\
\text { Video) }\end{array}$ & $03: 55$ & $x$ & Gil Green & & & 79 & Extrad & Sí & Grande & $\begin{array}{l}\text { Decorativa, } \\
\text { Sans serif }\end{array}$ \\
\hline $\begin{array}{l}\text { Liam Payne - Strip That } \\
\text { Down (Official Video) ft. } \\
\text { Quavo }\end{array}$ & $03: 41$ & $\mathrm{x}$ & & & Coreografía & & Extrad & No & Grande & Sans serif \\
\hline $\begin{array}{l}\text { Post Malone - rockstar ft. } \\
21 \text { Savage }\end{array}$ & 04:01 & & Emil Nava & & $x$ & 86 & Extrad & No & Pequeño & Modern \\
\hline
\end{tabular}


Pérez, J.P. / La visibilidad de la autoría en el videoclip post-televisivo:

La dignificación del vídeo musical a través de la reivindicación de los creadores en los créditos

\begin{tabular}{|c|c|c|c|c|c|c|c|c|c|c|}
\hline $\begin{array}{l}\text { Clean Bandit - Rockabye } \\
\text { ft. Sean Paul \& Anne-Marie } \\
\text { [Official Video] }\end{array}$ & 04:14 & $\mathrm{x}$ & & & & & Extrad & No & Grande & Sans serif \\
\hline $\begin{array}{l}\text { Calvin Harris - Feels } \\
\text { (Official Video) ft. Pharrell } \\
\text { Williams, Katy Perry, Big } \\
\text { Sean }\end{array}$ & $03: 42$ & $x$ & Emil Nava & $\begin{array}{l}\text { Productora, } \\
\text { Completo }\end{array}$ & & 86 & Extrad & Sí & Pequeño & Sans serif \\
\hline $\begin{array}{l}\text { Kygo, Selena Gomez - It } \\
\text { Ain't Me }\end{array}$ & 04:01 & & $\begin{array}{l}\text { Phillip R. } \\
\text { Lopez }\end{array}$ & & $x$ & 15 & Extrad & No & Pequeño & Oldstyle \\
\hline $\begin{array}{l}\text { Charlie Puth - Attention } \\
\text { [Official Video] }\end{array}$ & 03:51 & & (Emil Nava) & & $\mathrm{x}$ & (86) & & & & \\
\hline $\begin{array}{l}\text { Starley - Call On Me (Ryan } \\
\text { Riback Remix) }\end{array}$ & 03:17 & & $\begin{array}{l}\text { (Joseph } \\
\text { Toman) }\end{array}$ & & $\mathrm{x}$ & 7 & & & & \\
\hline $\begin{array}{l}\text { Maggie Lindemann - Pretty } \\
\text { Girl [Official Music Video] }\end{array}$ & 03:08 & & $\begin{array}{l}\text { (Roman } \\
\text { White) }\end{array}$ & & $\mathrm{x}$ & (46) & & & & \\
\hline $\begin{array}{l}\text { The Chainsmokers - Paris } \\
\text { (Video) }\end{array}$ & $03: 42$ & & $\begin{array}{l}\text { (Tomás } \\
\text { Whitmore) }\end{array}$ & & $x$ & (16) & & & & \\
\hline Julia Michaels - Issues & 03:02 & & $\begin{array}{l}\text { (Tabitha } \\
\text { Denholm) }\end{array}$ & & $x$ & (29) & & & & \\
\hline $\begin{array}{l}\text { Bruno Mars - That's What I } \\
\text { Like [Official Video] }\end{array}$ & $03: 30$ & $x$ & $\begin{array}{l}\text { (Bruno Mars } \\
\text { y Jonathan } \\
\text { Lia) }\end{array}$ & & $\mathrm{x}$ & $(8$ y 8$)$ & Extrad & No & Grande & Decorativa \\
\hline $\begin{array}{l}\text { Zedd, Alessia Cara - Stay } \\
\text { (Official Music Video }\end{array}$ & 03:33 & & (Tim Mattia) & & $\mathrm{x}$ & (47) & & & & \\
\hline $\begin{array}{l}\text { Ariana Grande - One Last } \\
\text { Time (Official) }\end{array}$ & 04:09 & $x$ & Max Landis & & & 1 & Extrad & No & Grande & Decorativa \\
\hline $\begin{array}{l}\text { Sam Smith - Too Good At } \\
\text { Goodbyes (Official Video) }\end{array}$ & $04: 24$ & $x$ & $\begin{array}{l}\text { Luke } \\
\text { Monaghan }\end{array}$ & & $x$ & 13 & Extrad & No & Pequeño & Modern \\
\hline $\begin{array}{l}\text { Anne-Marie - Ciao Adios } \\
\text { [Official Video] }\end{array}$ & 03:29 & $x$ & & & & & Extrad & No & Grande & Sans serif \\
\hline
\end{tabular}

La tabla recoge, tras la información del título de la pieza en YouTube y su duración, un apartado relativo a la visibilidad de la autoría en la muestra. Se han identificado con textos extradiegéticos en las piezas los nombres de los intérpretes en 22 clips (marcados con una equis). Las personas responsables de la dirección son identificadas en 24 casos; hemos puesto entre paréntesis aquellos nombres que aparecen en la "información contextual" de la página de YouTube pero no en el propio vídeo, contabilizando ocho casos. En 16 piezas el nombre del realizador aparecía en el vídeo como texto extradiegético. En la casilla "Personal/ Producción" hemos indicado otras formas de autoría al margen de la dirección identificadas (productora, actrices, coreógrafos, etc.). La siguiente columna recoge el número de videoclip de cada director o directora identificados. 
En cuanto al análisis tipográfico, observamos que los textos son extradiegéticos en todos los vídeos y solo aparecen dos nombres como texto diegético, de manera simultánea a los extradiegéticos. La tipografía es cinética en seis piezas y no lo es en las 18 restantes. El tamaño de los textos según las escalas que establecimos es grande en 14 vídeos, medio en cinco y pequeño en otros 5. Considerando el total de tipografías presentes (30), encontramos 15 ejemplos de tipografías Sans serif, seis decorativas, cuatro Modern, tres Script y dos Oldstyle.

\section{Resultados y discusión}

El análisis de la presencia de información sobre la autoría de las piezas analizadas permite advertir prácticas frecuentes que podrían ser representativas del estado actual del vídeo musical, incluso si los resultados son variados. Así, advertimos que de los 40 vídeos incluidos en el chart de ventas y popularidad de Reino Unido en 2017, 31 de ellos (el 77,5\%) recogen algún tipo de información en el propio discurso (como textos extradiegéticos) o en la "información contextual" de la página de YouTube.

En cuanto a los códigos textuales y gráficos relativos al reconocimiento de la autoría del vídeo presentes en el propio discurso, descubrimos lo que parece un cambio importante en las prácticas previas que ocultaban por completo la responsabilidad en la creación de dichas piezas. Así, en un $40 \%$ de los vídeos analizados (esto es, 16 de las 40 piezas) encontramos elementos gráficos y textuales que "firman" la autoría del clip. Aunque no se trata de una tendencia mayoritaria, sí podríamos en todo caso identificar un primer paso en el reconocimiento de la autoría en el propio discurso, como en los clásicos títulos de créditos de otros productos audiovisuales. Podemos interpretar que se está reivindicando la autoría del videoclip y que existe una intención por dar protagonismo a sus creadores, o por crear marcas de autoría en torno a determinados profesionales. 
Los códigos textuales extradiegéticos recogen el nombre de los intérpretes en un $55 \%$ de la muestra (22 videoclips). Se mantiene así la clásica atribución de la autoría del videoclip al intérprete, en una práctica que realmente poco aporta a la identidad de la autoría del resto de creadores, dado que la identificación del nombre del cantante o grupo ya era una práctica previa. Entendemos que su objetivo es identificar y así promocionar a aquellos en el caso de que la reproducción del vídeo se haga a través de plataformas o redes sociales que podrían prescindir de cualquier otro tipo de contenido textual. Incluso si perviven los canales de televisión temática especializados en videoclips, que ofrecían a través de recursos de grafismo el nombre de los intérpretes, la tendencia a "firmar" las piezas obedece a prácticas de reproducción de vídeo online y multiplataforma.

Las indicaciones en el discurso a aspectos relativos a la productora o estudio responsable de la producción aparece en sólo tres ocasiones (los clips de French Montana, Camila Cabello y Calvin Harris), lo que conformaría un 7,5\% de la muestra. En los tres casos la mención a la productora a través de textos extradiegéticos no es aislada y aparecen referidos también tanto el intérprete como la persona responsable de la dirección del videoclip. Se asume así un modelo de títulos de crédito similar al de las producciones cinematográficas o televisivas.

La mención a personal creativo de otras áreas artísticas relativas a la producción del videoclip aparece en un $20 \%$ de la muestra (8 videoclips), con mención sobre todo a actores y actrices (casos de los vídeos de Ed Sheeran o Shawn Mendes), pero también de la coreografía (videoclip de Martin Jensen), operador de cámara (Ed Sheeran) y otros aspectos relativos a la producción (como en el caso del clip de DJ Khaled, erigido como productor ejecutivo del videoclip).

Como caso excepcional, encontramos la pieza de Calvin Harris "Feels", que presenta una completa relación del personal involucrado en la creación 
del videoclip a través de una secuencia de créditos final similar a la de los largometrajes.

La asociación de los vídeos integrados en la plataforma de YouTube a una dirección URL permanente y la posibilidad de "información contextual" en dicha página web permiten añadir información adicional, enlaces para la compra o descarga digital del tema musical, la letra de la canción, comentarios personales del intérprete y, en un 35\% de los vídeos de la muestra (14 clips), el nombre de la persona responsable de la dirección.

La atención al modo en que los créditos recogen la atribución de la autoría permite constatar la amplia variedad de recursos y opciones dispuestas, si bien la funcionalidad en la composición tipográfica se impone por encima de otras aplicaciones más creativas. Frente a la riqueza creativa de muchos títulos cinematográficos (apuntemos al caso paradigmático de Saul y Elaine Bass), los créditos de la muestra analizada priman una carácter funcional e informativo.

Todos los textos son extradiegéticos, salvo en dos casos que además suponen referencias meta-cinematográficas: el nombre de DJ Khaled aparece bordado en una silla de director y el de Camila Cabello se muestra en la fachada de una sala de cine en la que entra la intérprete.

La legibilidad y la consecuente funcionalidad informativa se mejora con la ausencia de tipografías cinéticas (como ocurre en el 75\% de la muestra), y con las tipografías Sans serif, como tipos actuales, neutros y de excelente legibilidad en pantalla. Además, el tamaño de los textos es grande en el 58\% de los casos, exponiendo así la intención de búsqueda de la mejor legibilidad y de otorgar relieve a los textos. Las opciones más arriesgadas son minoritarias: un $20 \%$ de los textos son pequeños y un $25 \%$ de las tipografías son cinéticas. 
En la temprana tendencia identificada de inclusión de títulos de créditos en los videoclips prevalece el carácter informativo y la mejora de la legibilidad. Como ya ocurrió en el cine y en la ficción televisiva, podemos imaginar una evolución futura hacia planteamientos más creativos que aprovechen la enorme fuerza expresiva de los títulos de créditos y su potencialidad artística.

En la búsqueda de una relación entre la visibilidad de la autoría de la dirección del vídeo musical y el número de videoclips dirigidos por dichos realizadores, encontramos que generalmente aquellos nombres visibilizados contarán con una amplia trayectoria en la realización de videoclips, aspecto que nos podría llevar a concluir que la aparición del nombre del director en el discurso o en la "información contextual" de la página de YouTube responde a la propia celebridad del realizador.

Podríamos extrapolar esta conclusión al conjunto de la producción videográfica "firmada" por las personas responsables de la realización para interpretar que, de la misma forma que en los años noventa se detectó una generación de autores-clip, podría reconocerse una nueva generación de realizadores notables en el formato convertidos en nuevas celebridades o nuevos autoresclip. Dichos autores han desarrollado buena parte de su actividad dentro del videoclip, empresa alternada con la dirección de spots publicitarios y, en menor número de ocasiones, realización de producciones cinematográficas o televisivas. Se trataría, por lo tanto, de una generación de autores-clips para los que el formato musical comercial no parece suponer un medio de transición hacia formatos "mayores", sino una actividad en la que han logrado un prestigio certificado por su trabajo para los intérpretes musicales más comerciales del momento y las principales discográficas. La notoriedad de estas piezas, el alto número de ventas y reproducciones en plataformas online y la asociación a marcas, discográficas e intérpretes de éxito internacional parecen así prolongar la actividad de esta nueva generación de directores estrella del videoclip, 
ofreciendo un producto eficaz en el logro de sus objetivos comerciales y artísticos.

Destacaremos entre los directores "visibilizados" ocho nombres presentes entre los 31 vídeos analizados del chart tomado como muestra: Director X, con 210 vídeos musicales (Drake, Rihanna, Little Mix, Nelly Furtado, Iggy Azalea), según la base de datos Imvdb.com, es uno de los grandes nombres de la realización de videoclips actual; Dave Meyers, con base en California, destaca entre la actual generación de directores de vídeos musicales con 172 (Taylor Swift, Billie Eilish, Britney Spears, Ariana Grande, Pink, Jay Z, Katy Perry, Santana, Celine Dion, Justin Timberlake, Janelle Monáe), según Imvdb. com; Colin Tilley, con 137 videoclips (Justin Timberlake, Kendrick Lamar, Justin Bieber, Rihanna, Chris Brown, Nicki Minaj, Enrique Iglesias, Britney Spears, Iggy Azalea), según Imvdb.com; Emil Nava, director de al menos 92 videoclips (Calvin Harris, Ed Sheeran, Jennifer Lopez, Ellie Goulding, Rita Ora, Zara Larsson, Camila Cabello, Charlie Puth), según Imvdb.com (más de cien según otras fuentes); Gil Green, con base en Miami, es el realizador de al menos 79 vídeos (Jason Derulo, Pitbull, DJ Khaled, Akon, Birdman, Trey Songz, John Legend), según Imvdb.com; Eif Rivera, especializado en hip hop, cuenta con al menos con 57 videoclips (DJ Khaled, Cardi B, 50 cent, Ludacris), según Imvbd. com; Nicolas Tobias Følsgaard, director danés especializado en dirección de fotografía y con una carrera paralela como fotógrafo, ha dirigido al menos 49 piezas según recoge su página web; Tim Mattia, con base en Londres, firma al menos 49 videoclips (Hurts, The Killers, Zedd, The 1975), según Imvdb.com.

La mención a estos autores resulta por fuerza insuficiente al quedar a un lado directores y directoras convertidos prácticamente en celebridades dentro del formato, además de jóvenes promesas que han sorprendido a los aficionados al clip. En cualquier caso, como ocurrió en el campo del largometraje, somos ya capaces de identificar un acercamiento al videoclip consciente de su 
historia y trayectoria previa, en un ejercicio de autorreferencialidad que resulta inevitable en la actual escena de nostalgia y recuperación de cualquier tipo de producción cultural del pasado.

Una vez llegados a estos resultados, podemos interpretar cuáles creemos que son las razones por las que la autoría comienza a tener mayor visibilidad en el vídeo musical y sus consecuencias. En primer lugar, creemos que los títulos de crédito donde se recoge la identidad de sus creadores resultan de la mayor autonomía del videoclip con respecto a la televisión, autonomía que además refuerzan. Los vídeos musicales ya no necesitan el grafismo que integraba la televisión lineal. Incluso si, por lo general, estos contenidos se distribuyen desde plataformas (como YouTube) que titulan la pieza, la integración de la autoría en el propio discurso lo "firma", con independencia del modo de acceso al vídeo y de la forma de consumo.

Debemos matizar, en todo caso, esta autonomía del videoclip. Hemos comentado la distribución independiente a la televisión lineal a la programación y edición de los canales y que esa "libertad" permite el uso de recursos creativos y una posible mayor experimentación. Sin embargo, uno de los aspectos más directamente relacionados con la autonomía como puede ser la duración de las piezas, no se ve excesivamente afectado: de la muestra tomada, solo dos videoclips tienen una duración superior a los cinco minutos y la mayor parte ronda los 3-4 minutos. Incluso si la duración de estos vídeos es ligeramente superior a la de los sencillos en los que se basan, no se arriesga demasiado, lo que podría cuestionar la autonomía con respecto a la televisión lineal.

Podríamos concluir, en segundo lugar, que la visibilidad de la autoría contribuye a crear una noción de marca y de identidad en torno a los realizadores, los intérpretes, las productoras, etc. Aun tratándose de trabajos colectivos con muchas personas implicadas, el videoclip resulta así una suerte de formato que 
hibrida las marcas de los creadores. Por poner un ejemplo, en "Havana" de Camila Cabello, dirigido por el célebre Dave Meyers, convergen la identidad como estrella de la cantante cubana con la perspectiva creativa (la marca o el estilo, en definitiva) de Meyers (Vernallis, 2013).

En tercer y último lugar, podríamos interpretar que la visibilidad de la autoría dignifica el formato. Así, deja de ser un formato menor desde el momento en que se reivindica la autoría, una de los rasgos definidores de la producción cultural.

La visibilidad de la autoría permite al formato una consideración más cercana a la del producto cultural o a la creación artística, disimulando su función comercial. El videoclip no abandona dicho objetivo comercial o promocional, ligado a su propia esencia como formato, pero potencia su naturaleza de creación artística. La reivindicación de la autoría podría ser una estrategia también comercial para dotarlo de valor como producto cultural. No olvidemos que la generación de los autores-clip de los noventa aportó prestigio al formato y este salió reforzado desde la perspectiva crítica y académica (basta consultar los trabajos citados de Fidler, Hanson o Rodríguez-López).

\section{Conclusiones}

Los resultados de esta investigación pueden confirmar una apertura del concepto "autor-clip" hacia nuevos nombres, más allá de los directores estrella de los años noventa. Estos nuevos autores han desarrollado buena parte de su obra en un contexto de esplendor del formato, desde el momento en que las plataformas de vídeo online han contado con estas piezas como unos de sus contenidos con mayor demanda y mayor consumo, a tenor del número de reproducciones que adquieren en relación con otro tipo de contenidos. 
La prolífica actividad de algunos de estos realizadores, la posibilidad de transmisión de información que ha permitido la web y las bases de datos online como Imvdb.com y el reconocimiento de la videografía de algunos creadores por parte de crítica especializada y de aficionados al videoclip, han conducido a un contexto en el que la autoría de las piezas no sólo se ha visibilizado, sino que ha elevado a algunos de aquellos autores a la categoría de estrellas dentro del formato.

Aunque sólo algo más de la tercera parte de la muestra analizada incluía textos extradiegéticos con el nombre de la persona responsable de la dirección, este hecho supone un cambio notable con respecto a prácticas anteriores, en las que la autoría quedaba oculta y simbólicamente era atribuida al intérprete musical. Consideramos que, a la vista de las opciones permitidas por la distribución online de videoclips, irá en aumento la tendencia por otorgar mayor protagonismo y visibilidad a los autores de todo tipo en el área de la creación audiovisual, al punto de que los vídeos musicales con títulos de crédito completos podrían dejar de ser la excepción y convertirse en una práctica frecuente. Reivindicamos igualmente este aumento de la visibilidad de la autoría del vídeo musical desde el momento en que creemos que dignifica al formato y lo eleva a la posición en la que se encuentran otros formatos.

El reconocimiento de la autoría en el videoclip mediante textos con una intención más informativa y funcional que creativa lo equipara a otros tipos de producciones culturales en los que el prestigio viene ligado a la autoría y a la identificación del personal creativo. La consecuencia es la potenciación del carácter artístico del videoclip, sin que ello signifique un conflicto con su naturaleza como formato comercial o promocional. En definitiva, la autoría como marca de creación y como huella de estilo conduce a una mejora de su valor como producto cultural. 
Proponemos para una ampliación de esta investigación una atención más detallada a los realizadores y las realizadoras tanto de reconocido prestigio como noveles, por cuanto podría intentar identificarse una relación entre la notoriedad del director por la difusión de su trabajo y la visibilidad del autor manifestada a través de textos dentro del propio discurso audiovisual.

\section{Referencias}

Casetti, F. y Di Chio, F. (1991). Cómo analizar un film. Barcelona, España: Paidós.

De-Aguilera-Moyano, M., Castro-Higueras, A. y Pérez-Rufí, J.P. (2019). Entre broadcast yourself y broadcast whatever: la página de inicio de YouTube como síntesis de su estrategia empresarial. El profesional de la información, 28 (2), 1-14. doi: https://doi.org/10.3145/epi.2019.mar.06

Fidler, T. (2007). Music video auteurs: the directors label DVDs and the music videos of Chris Cunningham, Michel Gondry and Spike Jonze. (Ph.D Thesis). University of Western Australia. School of Social and Cultural Studies.

Hanson, M. (2006). Reinventing Music Video. Next-Generation Directors, Their Inspiration and Work. Mies, Suiza: Rotovision.

Higueras-Ruiz, M.J., Gómez-Pérez, F.J. y Alberich-Pascual, J. (2019). Revisión histórica y conceptual de la autoría y sus implicaciones en el medio televisivo: el concepto de autor en las series de televisión contemporáneas estadounidenses. Doxa Comunicación, 28, 79-96. doi: https://doi. org/10.31921/doxacom.n28a04

Keazor, H., and Wübbena; T (eds) (2008). Rewind, Play, Fast Forward. The Past, Present and Future of the Music Video. Estados Unidos, New Brunswick y Reino Unido, Londres: Transaction Publishers. 
Pérez-Rufí, J.P. (2017). El videoclip en Internet: cambios del formato en su distribución online. Razón y palabra, 21 (3), 574-605. Recuperado de https://bit.ly/2GEHqdD

Pérez-Rufí, J.P. (2018). Lyric vídeos: el encuentro del videoclip con el diseño gráfico y la composición tipográfica. Vivat Academia, 144, 1-18. doi: http:// doi.org/10.15178/va.2018.144.01-18

Reiss, S., and Feineman, N. (2000). Thirty Frames per second: The revolutionary art of the music video. Estados Unidos, Nueva York: Abrams.

Rodríguez-López, J. (2017). Videoclip de autor. El concepto de cine de autor y su aplicación al estilo del vídeo musical. Área Abierta, 17 (2), 229-240. doi: http://dx.doi.org/10.5209/ARAB.51717

Sedeño, A. (2010). Videoclips musicales en su transición a la red: nuevos subgéneros y apropiaciones del formato. Razón y Palabra, 71. Recuperado de https://goo.gl/qnCZib

Sedeño, A. (2012). Producción social de videoclips. Fenómeno fandom y vídeo musical en crisis. Comunicación: Revista Internacional de Comunicación Audiovisual, Publicidad y Estudios Culturales, 10, 1224-1235. Recuperado de https://goo.gl/29FXpk

Sedeño-Valdellós, A.M., Rodríguez-López, J. y Roger-Acuña, S. (2016). El videoclip postelevisivo actual. Propuesta metodológica y análisis estético. Revista Latina de Comunicación Social, 71, 332-348. doi: http://dx.doi. org/10.4185/RLCS-2016-1098

Selva-Ruiz, D. (2012). Nuevos fenómenos en el entorno online. La difusión del videoclip a través de Internet. Telos, 93. Recuperado de https://goo.gl/ bS28WM

Subires-Mancera, M.I. (2012). El fenómeno del lipdub como experiencia audiovisual colaborativa en la era de la web social. Comunicación: 
Revista Internacional de Comunicación Audiovisual, Publicidad y Estudios Culturales, 10, 1610-1620. Recuperado de https://goo.gl/8X23hh

Tarín-Cañadas, M. (2013). El videoclip interactivo como nuevo formato en la red. En B. Lloves Sobrado \& F. Segado Boj (Ed.), I Congreso Internacional de Comunicación y Sociedad Digital. Universidad Internacional de La Rioja, Logroño. Recuperado de https://goo.gl/YJjkc8

The Official Charts United Kingdom (2018). The Top 40 biggest songs of 2017 on the Official Chart. Officialcharts.com. Recuperado de https://goo.gl/ jHXWSh

Vernallis, C. (2008). "The Most Terrific Sandbox": Music Video Directors, Style, and the Question of the Auteur. Quarterly Review of Film and Video, 25 (5), 404-425. doi: https://doi.org/10.1080/10509200601093330

Vernallis, C. (2013). Unruly Media. YouTube, Music Video, and the New Digital Cinema. Oxford, Reino Unido: Oxford University Press. doi: https://doi. org/10.1093/acprof:oso/9780199766994.001.0001

Viñuela-Suárez, E. (2008). La autoría en el vídeo musical: signo de identidad y estrategia comercial. Revista Garoza, 8, 235-247. Recuperado de https:// goo.gl/Tc9KWb

Viñuela-Suárez, E. (2013). El videoclip del siglo XXI: el consumo musical de la televisión a Internet. Musiker, 20, 167-185. Recuperado de https://goo. gl/SyM8go

Williams, R. (2015). Diseño gráfico: principios y tipografía. Madrid, España: Anaya Multimedia.

Como citar: Pérez, J.P. (2020). La visibilidad de la autoría en el videoclip post-televisivo: la dignificación del vídeo musical a través de la reivindicación de los creadores en los créditos. Revista KEPES, 17 (21), 145-170. DOI: 10.17151/kepes.2020.17.21.6 